\title{
LOCAL COMPACTNESS IN RIGHT BOUNDED ASYMMETRIC NORMED SPACES
}

\author{
N. JONARD-PÉREZ AND E.A. SÁNCHEZ-PÉREZ
}

\begin{abstract}
We characterize the finite dimensional asymmetric normed spaces which are right bounded and the relation of this property with the natural compactness properties of the unit ball, as compactness and strong compactness. In contrast with some results found in the existing literature, we show that not all right bounded asymmetric norms have compact closed balls. We also prove that there are finite dimensional asymmetric normed spaces that satisfy that the closed unit ball is compact, but not strongly compact, closing in this way an open question on the topology of finite dimensional asymmetric normed spaces. In the positive direction, we will prove that a finite dimensional asymmetric normed space is strongly locally compact if and only if it is right bounded.
\end{abstract}

\section{INTRODUCTION}

It is well known that a normed vector space is locally compact if and only if it is finite dimensional. However, in the asymmetric case this is no longer true. In the context of asymmetric normed spaces, this matter is related to the relevant notion of right boundedness that has been widely used (see e.g. [2, 9]; see Section 22.1 for the definition). Right bounded asymmetric normed spaces were introduced in [14, Definition 16]. In that same paper it was stated that the unitary closed ball of a right bounded asymmetric normed space is always compact ([14, Proposition 17]). However, we will show in Example 4.1 that - except if the constant $r$ in the definition of right boundedness is $1-$ this result is not true. Therefore, it is natural to ask if it is possible to give a characterization of right boundedness for finite dimensional asymmetric normed spaces in terms of a weaker compactnesstype property: local compactness. Recall that a topological space $X$ is locally compact iff every point $x \in X$ has a local base consisting of compact neighborhoods.

The aim of this work is to solve that problem. Another relevant compactness property that can also be found in the literature - strong compactness-,

2010 Mathematics Subject Classification. 46A50, 46A55, 46B50, 52A07, 52A10.

Key words and phrases. Asymmetric norm, Right bounded, Compactness, Local compactness, Convex set.

The first author has been supported by Conacyt grant 252849 (México) and by PAPIIT grant IA104816 (UNAM, México). The second author has been supported by Ministerio de Economía y Competitividad (Spain) (project MTM2016-77054-C2-1-P). 
and its local version will be considered. As part of our main result, we will prove that right boundedness and strong local compactness are equivalent notions in the class of asymmetric normed spaces. However, we will also show that there are finite dimensional asymmetric normed spaces of dimension 3 such that the unitary closed ball is compact and right bounded, but not strongly compact (see Example 5.1). We will also prove other equivalences related with the geometry and the topology of the asymmetric unitary closed balls.

\section{Preliminaries}

Consider a real linear space $X$ and let $\mathbb{R}^{+}$be the set of non-negative real numbers. An asymmetric norm $q$ on $X$ is a function $q: X \rightarrow \mathbb{R}^{+}$such that (1) $q(a x)=a q(x)$ for every $x \in X$ and $a \in \mathbb{R}^{+}$,

(2) $q(x+y) \leq q(x)+q(y), x, y \in X$, and

(3) for every $x \in X$, if $q(x)=q(-x)=0$, then $x=0$.

The pair $(X, q)$ is called an asymmetric normed linear space. An asymmetric norm defines a non-symmetric topology on $X$ that is given by the open balls $B_{\varepsilon}^{q}(x):=\{y \in X: q(y-x)<\epsilon\}$. This topology is denoted by $\tau_{q}$.

We will denote by $\theta_{q}$ the set of all $x \in X$ such that $q(x)=0$. The set $\theta_{q}$ is a convex cone; this means that $\mu x \in \theta_{q}$ and $x+y \in \theta_{q}$ for every $x, y \in \theta_{q}$ and $\mu \geq 0$. This set plays a fundamental role in many topological, geometric and analytic results about asymmetric normed linear spaces. In particular, $(X, q)$ is $T_{1}$ if and only if $\theta_{q}=\{0\}$.

The following are well known results concerning the set $\theta_{q}$ (see [14 for the proofs) that will be needed in the paper.

(O1) For any open set $U \subset X$, we always have that $U=U+\theta_{q}$.

(O2) $K \subset X$ is compact if and only if $K+\theta_{q}$ is compact.

For every asymmetric normed space $(X, q)$ the map $q^{s}: X \rightarrow \mathbb{R}^{+}$defined by the rule

$$
q^{s}(x):=\max \{q(x), q(-x)\}, \quad x \in X,
$$

is a norm that generates a topology stronger than the one generated by $q$. We will use the symbols $B_{\varepsilon}^{q}$ and $B_{\varepsilon}^{q^{s}}$ to distinguish the balls of $(X, q)$ and $\left(X, q^{s}\right)$, respectively. More precisely, for every $x \in X$ and $\varepsilon>0$ we will use the following notations

$$
\begin{aligned}
& B_{\varepsilon}^{q}(x)=\{y \in X \mid q(y-x)<\varepsilon\}, \\
& B_{\varepsilon}^{q}[x]=\{y \in X \mid q(y-x) \leq \varepsilon\}, \\
& B_{\varepsilon}^{q^{s}}(x)=\left\{y \in X \mid q^{s}(y-x)<\varepsilon\right\}, \\
& B_{\varepsilon}^{q^{s}}[x]=\left\{y \in X \mid q^{s}(y-x) \leq \varepsilon\right\} .
\end{aligned}
$$

The set $B_{\varepsilon}^{q}[x]$ is called the closed ball of radius $\varepsilon$ centered at $x$. However, in general this set is not closed with respect to $\tau_{q}$. 
In order to avoid confusion, when necessary, we will say that a set is $q$ compact ( $q^{s}$-compact) if it is compact in the topology generated by $q\left(q^{s}\right)$. We will use the expressions $q$-open and $q$-closed sets $\left(q^{s}\right.$-open and $q^{s}$-closed sets) in the same way.

A subset $K \subset X$ in an asymmetric normed space $(X, q)$ is strongly $q$ compact (or simply, strongly compact) if and only if there exists a $q^{s}$-compact set $S \subset X$ such that

$$
S \subset K \subset S+\theta_{q} .
$$

Every strongly compact set is $q$-compact, but the converse is not true (see e.g. 2, 8, 15]). If each point of the asymmetric normed space $X$ has a local base consisting of strongly compact sets we will say that $X$ is strongly locally compact. Evidently, if $X$ is strongly locally compact then it is also locally compact.

Since $q(x) \leq q^{s}(x)$ for every $x \in X$, we always have the following contentions

$$
B_{\varepsilon}^{q^{s}}(x)+\theta_{q} \subset B_{\varepsilon}^{q}(x) \quad \text { and } \quad B_{\varepsilon}^{q^{s}}[x]+\theta_{q} \subset B_{\varepsilon}^{q}[x] .
$$

Addition in asymmetric normed spaces is always continuous but the scalar multiplication is not (see e.g. 7]). However it is well-known that multiplication by non negative scalars is continuous. For the convenience of the reader, we include the proof of this result that will be used often in the paper.

Lemma 2.1. Let $(X, q)$ be an asymmetric normed space. The map $\mathbb{R}^{+} \times X$ given by $(\mu, x) \rightarrow \mu x$ is continuous if we endow $\mathbb{R}^{+}$with the Euclidean topology.

Proof. Let $\varepsilon>0$ and $(\mu, x) \in \mathbb{R}^{+} \times X$. Then there exists $\delta_{1}>0$ such that $\delta_{1} q^{s}(x)<\varepsilon / 2$. Since $\mu \geq 0$, if we define $\delta_{2}:=\varepsilon / 2\left(\mu+\delta_{1}\right)$ we have that $\delta_{2}>0$. Now take $\lambda \geq 0$ such that $|\lambda-\mu|<\delta_{1}$ and $y \in B_{\delta_{2}}^{q}(x)$. Thus

$$
\begin{aligned}
q(\lambda y-\mu x) & =q(\lambda y-\lambda x+\lambda x-\mu x) \\
& \leq q(\lambda y-\lambda x)+q(\lambda x-\mu x) \\
& \leq \lambda q(y-x)+q^{s}(\lambda x-\mu x) \\
& <\left(\mu+\delta_{1}\right) \frac{\varepsilon}{2\left(\mu+\delta_{1}\right)}+|\lambda-\mu| q^{s}(x) \\
& <\frac{\varepsilon}{2}+\delta_{1} q^{s}(x)<\frac{\varepsilon}{2}+\frac{\varepsilon}{2}=\varepsilon .
\end{aligned}
$$

This proves the lemma.

2.1. Equivalent and right bounded asymmetric norms. Let us show some basic facts on the relation among equivalent asymmetric norms and the right boundedness property for the corresponding spaces that will be used later on. Some of the proofs are straightforward, so we will write only some hints for getting them. 
Let $X$ be a real vector space. Two asymmetric norms in $X, q$ and $p$, are said to be equivalent if and only if there exist $\kappa>0$ and $\lambda>0$ such that

$$
\kappa q(x) \leq p(x) \leq \lambda q(x) \quad \text { for all } x \in X,
$$

(or equivalently, if and only if $\lambda B_{1}^{q}[0] \subset B_{1}^{p}[0] \subset \kappa B_{1}^{q}[0]$ ). Obviously, two equivalent asymmetric norms in $X$ generate the same topology. For the proof of the next result it is enough to take into account that for equivalent $q$ and $p, q(x)=0$ if and only if $p(x)=0$.

Lemma 2.2. Let $q$ and $p$ be equivalent asymmetric norms in a vector space $X$. Then $\theta_{p}=\theta_{q}$.

An asymmetric norm $q$ in a vector space $X$ is said to be right bounded if and only if there exists $r>0$ such that

$$
r B_{1}^{q}[0] \subset B_{1}^{q^{s}}[0]+\theta_{q} .
$$

In this case we also say that $B_{1}^{q}[0]$ is right bounded. In the particular case when $r=1$, we will simply say that $q$ is 1 -bounded. Using (1) we infer the following

Remark 2.3. Let $(X, q)$ be an asymmetric normed space. The norm $q$ is 1-bounded if and only if $B_{1}^{q}[0]=B_{1}^{q^{s}}[0]+\theta_{p}$.

Lemma 2.4. An asymmetric normed space $(X, q)$ is right bounded if and only if there exists a $q^{s}$-bounded set $K$ such that

$$
B_{1}^{q}[0] \subset K+\theta_{q}
$$

Proof. If $(X, q)$ is right bounded, we can find $r>0$ such that $r B_{1}^{q}[0] \subset$ $B_{1}^{q^{s}}[0]+\theta_{q}$. Then the set $K:=\frac{1}{r} B_{1}^{q^{s}}[0]$ is $q^{s}$-bounded and

$$
B_{1}^{q}[0] \subset K+1 / r \theta_{q}=K+\theta_{q} .
$$

For the second implication, let $h>0$ be such that $K \subset h B_{1}^{q^{s}}[0]$. Then

$$
B_{1}^{q}[0] \subset K+\theta_{q} \subset h B_{1}^{q^{s}}[0]+\theta_{q}=h B_{1}^{q^{s}}[0]+h \theta_{q} .
$$

Thus $1 / h B_{1}^{q}[0] \subset B^{q^{s}}+\theta_{q}$, which proves that $q$ is right bounded.

Lemma 2.5. Let $q$ and $p$ be equivalent asymmetric norms in a finite dimensional vector space $X$. Then $q$ is right bounded if and only if $p$ is right bounded.

Proof. Let us assume that $q$ is right bounded. Since $X$ is finite dimensional, the norms $q^{s}$ and $p^{s}$ are equivalent. Then there exists a positive number $\kappa$ such that $B_{1}^{q^{s}}[0] \subset \kappa B_{1}^{p^{s}}[0]$. On the other hand, since $q$ is right bounded there exists $r^{\prime}>0$ with the property that $r^{\prime} B_{1}^{q}[0] \subset B_{1}^{q^{s}}[0]+\theta_{q}$. Finally, since $p$ and $q$ are equivalent, we can find $\lambda>0$ such that $\lambda B_{1}^{p}[0] \subset B_{1}^{q}[0]$. 
Now we can use all previous contentions and Lemma 2.2 to conclude that

$$
\begin{aligned}
r^{\prime} \lambda B_{1}^{p}[0] & \subset r^{\prime} B_{1}^{q}[0] \subset B_{1}^{q^{s}}[0]+\theta_{q} \\
& \subset \kappa B_{1}^{p^{s}}[0]+\theta_{q}=\kappa B_{1}^{p^{s}}[0]+\theta_{p} \\
& =\kappa B_{1}^{p^{s}}[0]+\kappa \theta_{p}=\kappa\left(B_{1}^{p^{s}}[0]+\theta_{p}\right) .
\end{aligned}
$$

Then we infer that $r B_{1}^{p}[0] \subset B_{1}^{p^{s}}[0]+\theta_{p}$ where $r=r^{\prime} \lambda / \kappa$. The other implication can be proven in a similar way.

2.2. The canonical 1-bounded equivalent asymmetric norm. In 9] Conradie introduced a new asymmetric norm associated to an asymmetric normed space $(X, p)$ in the following way. For every $x \in X$ let us define

$$
q_{p}(x)=\inf \left\{p^{s}(x-y) \mid y \in \theta_{p}\right\} .
$$

It was proven in $\left[9\right.$ that $q_{p}: X \rightarrow[0, \infty)$ is an asymmetric norm satisfying the following properties:

(p1) $p(x) \leq q_{p}(x)$ for all $x \in X$,

(p2) $B_{1}^{q_{p}}[0] \subset B_{1}^{p}[0]$

(p3) $B_{1}^{q_{p}}[0]=B_{1}^{p^{s}}[0]+\theta_{p}$,

(p4) $p$ is equivalent to $q_{p}$ if and only if $p$ is right bounded.

In the next lemma we prove some other fundamental properties about the norm $q_{p}$ that will be used later on.

Lemma 2.6. Let $(X, p)$ be an asymmetric normed space. Then the following statements hold

(p5) $\theta_{p}=\theta_{q_{p}}$ (equivalently, $p(x)=0$ if and only if $q_{p}(x)=0$ ).

(p6) $p^{s}=q_{p}^{s}$ (in particular $B_{1}^{p^{s}}[0]=B_{1}^{q_{p}^{s}}[0]$ ).

(p7) $q_{p}$ is 1-bounded.

(p8) $B_{1}^{q_{p}}[0]=B_{1}^{p}[0]$ if and only if $p$ is 1-bounded.

Proof. (p5) The inclusion $\theta_{q_{p}} \subset \theta_{p}$ follows from property (p1). Now, if $x \in \theta_{p}$, we can use the definition of $q_{p}$ to infer that

$$
0 \leq q_{p}(x) \leq p^{s}(x-x)=p^{s}(0)=0 .
$$

Then $q_{p}(x)=0$ and therefore $x \in \theta_{q_{p}}$.

(p6) Let $x \in X$. By property (p1), we have that $q_{p}(x) \geq p(x)$ and $q_{p}(-x) \geq p(-x)$. Thus

$$
q_{p}^{s}(x)=\max \left\{q_{p}(x), q_{p}(-x)\right\} \geq \max \{p(x), p(-x)\}=p^{s}(x) .
$$

On the other hand, the definition of $q_{p}$ guarantees that $p^{s}(x)=p^{s}(x-0) \geq$ $q_{p}(x)$ and $p^{s}(-x)=p^{s}(-x-0) \geq q_{p}(-x)$. Since $p^{s}$ is a norm, $p^{s}(x)=p^{s}(-x)$ and therefore

$$
p^{s}(x) \geq \max \left\{q_{p}(x), q_{p}(-x)\right\}=q_{p}^{s}(x) .
$$

Then we get $p^{s}(x)=q_{p}^{s}(x)$, as desired. 
(p7) Using Remark 2.3 and properties (p3), (p5) and (p6) we get that

$$
B_{1}^{q_{p}}[0]=B_{1}^{p^{s}}[0]+\theta_{p}=B_{1}^{q_{p}^{s}}[0]+\theta_{q_{p}} .
$$

Then $q_{p}$ is 1-bounded.

(p8) This is a direct consequence of Remark 2.3 and property (p3).

\section{Convexity and COMpactness}

In this section we will show some results relating to compactness properties of subsets of asymmetric normed spaces, mainly for convex sets. The notion of local compactness arises in a natural way in the characterizations, which are given in terms of an equivalent asymmetric norm satisfying some special properties.

It is well known that in finite dimensional normed spaces, the convex hull of a compact set is compact. In the following lemma we show the asymmetric version of this result.

Lemma 3.1. Let $A \subset X$ be a q-compact set in the finite dimensional asymmetric normed space $(X, q)$. Then the convex hull $\operatorname{conv}(A)$ is also q-compact.

Proof. Let $n$ be the (algebraic) dimension of $X$. We apply Carathéodory's Theorem ([18, Theorem 2.2.4]) to infer that

$$
\operatorname{conv}(A)=\left\{\sum_{i=0}^{n} \lambda_{i} a_{i}: a_{i} \in A, \lambda_{i} \geq 0 \text { and } \sum_{i=0}^{n} \lambda_{i}=1\right\} .
$$

On the other hand, the topological product $A^{n+1}$ is also compact since $A$ is compact. Now, let us consider the $n$-dimensional simplex

$$
\Delta=\left\{\left(\lambda_{0}, \ldots, \lambda_{n}\right) \in \mathbb{R}^{n+1} \mid \lambda_{i} \geq 0 \text { and } \sum_{i=0}^{n} \lambda_{i}=1\right\} .
$$

If we equip $\Delta$ with the Euclidean topology, it becomes a compact space. Now, let $\varphi: A^{n+1} \times \Delta \rightarrow \operatorname{conv}(A)$ be the natural map defined by

$$
\varphi\left(a_{0}, \ldots, a_{n}, \lambda_{0}, \ldots, \lambda_{n}\right)=\sum_{i=0}^{n} \lambda_{i} a_{i}
$$

Clearly $\varphi$ is an onto function. Now observe that $\varphi$ is continuous since the addition is continuous in $X$ ([7, Proposition 1.1.40]) and the scalar multiplication by positive numbers is also continuous (Lemma 2.1). Using the fact that $A^{n+1} \times \Delta$ is compact we can then conclude that $\operatorname{conv}(A)$ is also compact.

Lemma 3.2. Let $A$ be a strongly compact set in the finite dimensional asymmetric normed space $(X, q)$. Then $\operatorname{conv}(A)$ is also strongly compact. 
Proof. Let $K \subset A$ be a $q^{s}$-compact set such that $K \subset A \subset K+\theta_{q}$. By [18, Theorem 2.2.6], $\operatorname{conv}(K)$ is $q^{s}$-compact. Furthermore, we have that

$$
\operatorname{conv}(K) \subset \operatorname{conv}(A) \subset \operatorname{conv}\left(K+\theta_{q}\right) .
$$

Since $\operatorname{conv}(K)+\theta_{q}$ is a convex set containing $K+\theta_{q}$, we conclude that $\operatorname{conv}\left(K+\theta_{q}\right) \subset \operatorname{conv}(K)+\theta_{q}$ and therefore

$$
\operatorname{conv}(K) \subset \operatorname{conv}(A) \subset \operatorname{conv}(K)+\theta_{q} .
$$

This proves that $\operatorname{conv}(A)$ is strongly compact.

Lemma 3.3. Let $A$ be a strongly compact set in a finite dimensional asymmetric normed space $(X, q)$. Then the $q^{s}$-closure of $A$ is also strongly compact.

Proof. Let $K \subset X$ be a $q^{s}$-compact set such that $K \subset A \subset K+\theta_{q}$. For any $B \subset X$, let us denote by $\bar{B}^{s}$ the $q^{s}$-closure of $B$. Then we have that

$$
\bar{K}^{s} \subset \bar{A}^{s} \subset{\overline{K+\theta_{q}}}^{s} \text {. }
$$

Since $K$ is $q^{s}$-closed we have that $K=\bar{K}^{s}$. On the other hand, since $\theta_{q}$ is $q^{s}$-closed (see e.g. [16, Lemma 3.6]) by [18, Theorem 1.8.10-(ii)] the set $K+\theta_{q}$ is $q^{s}$-closed and therefore $K+\theta_{q}={\overline{K+\theta_{q}}}^{s}$. Then $K \subset \bar{A}^{s} \subset K+\theta_{q}$ which proves that $\bar{A}^{s}$ is strongly compact, as desired.

Proposition 3.4. Let $(X, q)$ be a finite dimensional asymmetric normed space. If the origin has a compact neighbourhood $U$, then there exists an equivalent asymmetric norm $p$ such that each closed ball $B_{r}^{p}[x]$ is compact. If additionally $U$ is strongly compact, then the balls $B_{r}^{p}[x]$ are also strongly compact.

Proof. Let $U$ be a $q$-compact neighbourhood of the origin. Then there exist $\varepsilon>0$ and $\kappa>0$ such that

$$
\varepsilon B_{1}^{q}[0] \subset U \subset \kappa B_{1}^{q}[0] .
$$

Let us denote by $A$ the $q^{s}$-closure of the convex hull of $U$. By Lemma 3.1 and [16, Lemma 3.2], we have that $A$ is compact. Since $\kappa B_{1}^{q}[0]$ is convex and $q^{s}$-closed, we have that

$$
\varepsilon B_{1}^{q}[0] \subset U \subset A \subset \kappa B_{1}^{q}[0]
$$

Now let $p$ be defined as the gauge functional of $A$. Namely,

$$
p(x):=\{\inf t>0 \mid x \in t A\} .
$$

Since $A$ is absorbent, convex and does not contain any line, $p$ defines an asymmetric norm such that $B_{1}^{p}[0]=A$ (see [7, 9]). Furthermore, by (2) $p$ is equivalent to $q$. Since $A$ is $q$-compact, it is also $p$-compact and all the closed balls $B_{r}^{p}[x]=r B_{1}^{p}[0]+r x$ are also compact.

Now, if $U$ is strongly compact we get by Lemma 3.3 that $A=B_{1}^{p}[0]$ is strongly compact and therefore all closed balls $B_{r}^{p}[x]$ are strongly compact, as desired. 


\section{Strong Local COMPACTNESS}

The aim of this section is to analyze the boundedness properties of strong locally compact asymmetric spaces. As we said in the Introduction, right bounded spaces have not necessarily compact unitary balls. Indeed, let us start with the following example, which is a counterexample to [14, Proposition 17].

Example 4.1. There exists a finite dimensional asymmetric normed space $(X, q)$ such that the unitary closed ball $B_{1}^{q}[0]$ is right bounded and non compact.

Proof. Let $X=\mathbb{R}^{2}$. Define the set $A \subset X$ as follows

$$
A=\left\{(x, y) \in \mathbb{R}^{2} \mid y \leq \frac{1}{x-2}+2, x<2\right\} .
$$

Let $q: X \rightarrow[0, \infty)$ be defined as the gauge functional of $A$. Namely

$$
q((x, y))=\inf \{t>0 \mid(x, y) \in t A\} .
$$

Clearly $q$ is an asymmetric norm in $X$ such that $A=B_{1}^{q}[(0,0)]$. Furthermore, it is easy to verify that $\theta_{q}=\{(x, y) \mid x \leq 0, y \leq 0\}$ and $B_{1}^{q}[(0,0)] \subset(2,2)+\theta_{q} \subset 2 B_{1}^{q^{s}}[(0,0)]+\theta_{q}$. Then we can conclude that

$$
\frac{1}{2} B_{1}^{q}[(0,0)] \subset B_{1}^{q^{s}}[(0,0)]+\theta_{q}
$$

and therefore $q$ is right bounded. Now let us observe that $B_{1}^{q}[(0,0)]$ is non compact. Indeed, let us consider the family $\mathcal{U}=\left\{B_{1}^{q}((2, t))\right\}_{t<0}$. Clearly, for every $t<0$,

$$
B_{1}^{q}((2, t))=\left\{(x, y) \in \mathbb{R}^{2}, y<\frac{1}{x-4}+2+t, x<4\right\}
$$

and $\mathcal{U}$ is an open cover for $B_{1}^{q}[(0,0)]$. Furhtermore, if $t>s$, then $B_{1}^{q}((2, s)) \subset$ $B_{1}^{q}((2, t))$ and therefore $\mathcal{U}$ is a nested family. A simple calculation shows that every point $(x, y) \in B_{1}^{q}[(0,0)]$ such that $y=1 /(x-2)+2$ and $x \geq$ $3-\sqrt{1-2 / t}$ cannot belong to $B_{1}^{q}((2, t))$. Since $\mathcal{U}$ is nested we conclude that $B_{1}^{q}[(0,0)]$ cannot be covered with a finite subfamily of $\mathcal{U}$ and therefore $B_{1}^{q}[(0,0)]$ is non compact.

Remark 4.2. The norm $q$ defined in Example 4.1 is equivalent to the asymmetric lattice norm $p: \mathbb{R}^{2} \rightarrow \mathbb{R}$ given by

$$
p((x, y))=\max \left\{x^{+}, y^{+}\right\}
$$

where $x^{+}=\max \{x, 0\}$. It is well known that $B_{1}^{p}[(0,0)]$ is $p$-compact and therefore $(X, p)$ is locally compact (in fact, it is strongly locally compact). Since $(X, p)$ is equivalent to $(X, q)$ we infer that an asymmetric normed space can be locally compact, even if the unitary closed ball is not compact. 
After Example 4.1 and Remark 4.2, it is natural to ask what is the relation between right boundedness and strong local compactness in finite dimensional asymmetric normed spaces. The aim of this section is to answer that question.

Lemma 4.3. Let $(X, q)$ be a finite dimensional asymmetric normed space. If $q$ is 1-bounded, then $B_{1}^{q}[0]$ is strongly compact and therefore $(X, q)$ is strongly locally compact.

Proof. Observe that $B_{1}^{q^{s}}[0]$ is $q^{s}$-compact (because $X$ is finite dimensional). Now, since $q$ is 1-bounded, we have that

$$
B_{1}^{q^{s}}[0] \subset B_{1}^{q}[0]=B_{1}^{q^{s}}[0]+\theta_{q} .
$$

This directly implies that $B_{1}^{q}[0]$ is strongly compact.

Many important asymmetric normed spaces are 1-bounded. For example, every asymmetric norm defined by a Banach lattice norm is 1-bounded ([2, Lemma 1]). Furthermore, as we have already shown, the norm $q_{p}$ associated to an asymmetric normed space $(X, p)$ is 1-bounded. This gives us the following

Corollary 4.4. If $(X, p)$ is a finite dimensional asymmetric normed space, then $B_{1}^{q_{p}}[0]$ is strongly compact.

Lemma 4.5. The asymmetric normed space $(X, p)$ is right bounded if and only if there exists an equivalent norm $q$ such that $q$ is 1-bounded.

Proof. For the first implication just consider $q=q_{p}$ and properties (p4) and (p7). The second implication follows directly from Lemma 2.5

Proposition 4.6. Let $(X, p)$ be a finite dimensional asymmetric normed space. If the origin has a strongly compact neighborhood, then $p$ is right bounded.

Proof. By Proposition 3.4, we can find an equivalent norm $q$ such that $B_{1}^{q}[0]$ is strongly compact. Then there exists a $q^{s}$-compact set $K \subset X$ such that

$$
K \subset B_{1}^{q}[0] \subset K+\theta_{q} .
$$

Since $X$ is finite dimensional we have that $q^{s}$ and $p^{s}$ are equivalent norms, then $K$ is $p^{s}$ compact and we can find $a>0$ such that $K \subset a B_{1}^{p^{s}}$. On the other hand, since $q$ is equivalent to $p$, there exists $b>0$ with $b B_{1}^{p}[0] \subset B_{1}^{q}[0]$. Using Lemma 2.2, we infer that

$$
\begin{aligned}
b B_{1}^{p}[0] & \subset B_{1}^{q}[0] \subset K+\theta_{q} \subset a B_{1}^{p^{s}}[0]+\theta_{q} \\
& =a B_{1}^{p^{s}}[0]+a \theta_{p}=a\left(B_{1}^{p^{s}}[0]+\theta_{p}\right) .
\end{aligned}
$$

Therefore $r B_{1}^{p}[0] \subset B_{1}^{p^{s}}[0]+\theta_{p}$ with $r=b / a$, and then $p$ is right bounded, as desired.

Now we summarize all the previous work in the main result of this section. 
Theorem 4.7. Let $(X, p)$ be a finite dimensional asymmetric normed space. The following statements are equivalent:

(1) $p$ is right bounded.

(2) There exists an equivalent asymmetric norm $q$ in $X$ such that $q$ is 1bounded.

(3) There exists an equivalent asymmetric norm $q$ in $X$ such that $B_{1}^{q}[0]$ is strongly compact.

(4) $(X, p)$ is strongly locally compact.

Proof. The part $(1) \Longleftrightarrow(2)$ was already proved in Lemma 4.5 .

Implication $(2) \Longrightarrow(3)$ follows from Lemma 4.3 .

Finally, implication $(3) \Longrightarrow(4)$ is obvious and $(4) \Longrightarrow(1)$ was proved in Proposition 4.6.

We finish this section by giving a useful characterization of equivalent right bounded asymmetric norms.

Theorem 4.8. Let $X$ be a real finite dimensional linear space. Two right bounded asymmetric norms $p$ and $p^{\prime}$ are equivalent if and only if $\theta_{p}=\theta_{p^{\prime}}$.

Proof. The "only if" implication was already proven in Lemma 2.2, For the "if" implication let $q:=q_{p}$ and $q^{\prime}:=q_{p^{\prime}}$. Then $q$ and $q^{\prime}$ are 1-bounded and therefore $B_{1}^{q}[0]=B_{1}^{q^{s}}[0]+\theta_{q}$ and $B_{1}^{q^{\prime}}[0]=B_{1}^{q^{\prime s}}[0]+\theta_{q}^{\prime}$.

On the other hand, since $X$ is finite dimensional, the norms $q^{s}$ and $q^{\prime s}$ are equivalent and therefore we can find $\mu>0$ and $\lambda>0$ with the property that

$$
\mu B_{1}^{q^{s}}[0] \subset B_{1}^{q^{s}}[0] \subset \lambda B_{1}^{q^{s}}[0] .
$$

Since $\theta_{p}=\theta_{p^{\prime}}$ we can use property (p5) to infer that $\theta_{q}=\theta_{q^{\prime}}$. Thus

$$
\begin{aligned}
\mu B_{1}^{q}[0] & =\mu\left(B_{1}^{q^{s}}[0]+\theta_{q}\right)=\mu B_{1}^{q^{s}}[0]+\mu \theta_{q} \\
& =\mu B_{1}^{q^{s}}[0]+\theta_{q} \subset B_{1}^{q^{s}}[0]+\theta_{q} \\
& \subset \lambda B_{1}^{q^{s}}[0]+\theta_{q}=\lambda B_{1}^{q^{s}}[0]+\lambda \theta_{q} \\
& =\lambda\left(B_{1}^{q^{s}}[0]+\theta_{q}\right)=\lambda B_{1}^{q}[0] .
\end{aligned}
$$

Since $B_{1}^{q^{\prime s}}[0]+\theta_{q}=B_{1}^{q^{\prime s}}[0]+\theta_{q}^{\prime}=B_{1}^{q^{\prime}}[0]$, we conclude that $q$ and $q^{\prime}$ are equivalent. Now, since $p$ and $p^{\prime}$ are right bounded, they are equivalent to $q$ and $q^{\prime}$ (by property (p4)), respectively, and therefore $p$ and $p^{\prime}$ are also equivalent.

\section{Right Boundedness AND THE GEOMETRY OF THE UNITARY BALL}

Let us finish the paper by exploring the relation among right boundedness, compactness and the geometry of the unitary closed ball in a finite dimensional asymmetric normed space.

In 16 it was proven that the convex hull of the extreme points of a compact convex set in an asymmetric normed space defines the topology of 
the set. In this section we will use the techniques used there to understand the relation among the geometry and the topology of the unitary closed balls in finite dimensional asymmetric normed spaces.

Given a convex set $K$ in an asymmetric normed space $(X, q)$, let us denote by $E(K)$ the extreme points 1$]$ of $K+\theta_{q}$, and by $S(K)$ the convex hull of $E(K)$.

First, it is interesting to note compactness of the set $E(K)$ is not a necessary condition to guarantee the compactness of a convex set $K$. For example, consider the the asymmetric lattice norm $\left(\mathbb{R}^{2}, p\right)$ defined in Remark 4.2 . Then the set

$$
K=\left\{(x, y) \in \mathbb{R}^{2} \mid x \leq 0 \text { and } y \leq-x^{2}\right\}
$$

is a strongly compact convex set with an unbounded set $E(K)$.

On the other hand it was proven in [8] that in a finite dimensional asymmetric normed lattice $(X, q)$, all $q$-compact and $q^{s}$-closed sets are strongly compact. If the dimension of $X$ is 2 , then it is also known (see [15]) that every $q$-compact and convex set is strongly compact (even if it is not $q^{s}$ closed). Nevertheless, not all compact convex sets are strongly compact (the reader can find an example of this in [15]). After these results, it is natural to ask if in general, a compact convex set of a finite dimensional asymmetric normed space $(X, q)$ is strongly compact if it is $q^{s}$-closed. Even more, after Theorem 4.7 another natural question arises. On one hand, Theorem 4.7 shows that local compactness of an equivalent norm characterizes right boundedness and strong local compactness. Therefore, we can ask if in general compactness of the unit ball implies strong compactness. The next example solves these two questions negatively.

Example 5.1. There is a finite dimensional asymmetric normed space such that $B_{1}^{q}[0]$ is compact, but not strongly compact.

Proof. In order to see this, consider the cylinder $C=\left\{(x, y, z) \in \mathbb{R}^{3} \mid\right.$ $y^{2}+z^{2} \leq 1$ and $\left.x \leq 1\right\}$ in $\mathbb{R}^{3}$, and let us define

$$
R=\left\{(x, 0,0) \in \mathbb{R}^{3} \mid x \leq 0\right\} .
$$

Let $p: \mathbb{R}^{3} \rightarrow[0, \infty)$ be the gauge functional over the set $C$

$$
p(u)=\inf \{t \geq 0 \mid u \in t C\} .
$$

Then $p$ is an asymmetric norm in $\mathbb{R}^{3}$, for whom the unitary closed ball is the set $C$ and such that $\theta_{p}=R$. We will define an equivalent norm $q: \mathbb{R}^{3} \rightarrow[0, \infty)$ with the required characteristics.

To do that, let us start by defining, for each $n \in \mathbb{N}$, the values:

$$
y_{n}=\cos \left(\frac{n \pi}{2(n+1)}\right) \quad \text { and } \quad z_{n}=\sin \left(\frac{n \pi}{2(n+1)}\right) .
$$

\footnotetext{
1 A point $x \in A$ is an extreme point of $A$ if $x=y=z$ whenever $y, z \in A$ and $x=\lambda y+(1-\lambda) z$ for some $\lambda \in(0,1)$.
} 
And let us consider the sequence $\left(u_{n}\right)_{n \in \mathbb{N}}$ in $\mathbb{R}^{3}$, where $u_{n}=\left(-n, y_{n}, z_{n}\right)$. Evidently, in the topology $\tau_{p}$, the sequence $\left(u_{n}\right)_{n \in \mathbb{N}}$ converges to the point $(1,0,0)$, and therefore the set $A_{1}=\left\{u_{n} \mid n \in \mathbb{N}\right\} \cup\{(1,0,0)\}$ is p-compact.

On the other hand, the set $A_{2}=\left\{(1, y, z) \in \mathbb{R}^{3}|| y|+| z \mid \leq 1\right\}$ is clearly $p^{s}$-compact and therefore it is also $p$-compact. Thus $A_{1} \cup A_{2}$ is compact. Let $A=\operatorname{conv}\left(A_{1} \cup A_{2}\right)$. By Lemma 3.1. $A$ is compact and therefore $A+\theta_{p}$ is also compact (Property (O2)). Therefore we can use [16, Lemma 3.2] in order to conclude that $B:=\bar{A}^{s}$ is a $p^{s}$-closed $p$-compact convex set. Furthermore, the set $B$ satisfies

(1) $B=B+\theta_{p}$

(2) $\frac{1}{\sqrt{2}} C \subset B \subset C$

Define now $q: \mathbb{R}^{3} \rightarrow[0, \infty)$ as the gauge functional over $B$. Namely

$$
q(u)=\inf \{t \geq 0 \mid u \in t B\} .
$$

Clearly $B$ is the closed unitary ball with respect to $q$ and $\theta_{q}=R=\theta_{p}$. Property (2) implies that $q$ is equivalent to $p$. In particular, since $p$ is obviously right bounded, we also get that $q$ is right bounded (by Lemma 2.5). By the same reason, since $B$ is $p$-compact it is also $q$-compact, as desired.

It just remains to prove that $B$ is not strongly compact. For that purpose, suppose the opposite. Then we can find a $q^{s}$-compact set $K$ such that $K \subset B \subset K+\theta_{q}$. Since $B$ is convex, we can use Lemma 3.1 in order to assume without any loss of generality that the set $K$ is convex. We can also observe that

$$
K \subset B \subset K+\theta_{q} \subset B+\theta_{q}=B,
$$

and therefore $B=K+\theta_{q}$. Now, by [16, Theorem 4.1] the set of extreme points $E(B)$ is contained in $K$. Since every point in the set $A_{1}$ is an extreme point of $B$, we infer that $A_{1} \subset K$. In particular, we get that $A_{1}$ is $q^{S_{-}}$ bounded, which is a contradiction.

In particular this gives the existence of $q^{s}$-closed convex sets in finite dimensional asymmetric normed spaces that are compact but not strongly compact (c.f. [8]). Example 5.1 also shows that there is a compact unitary closed ball of an asymmetric normed space of dimension 3 such that the set of its extreme points $E\left(B_{1}^{q}[0]\right)$ is not $q^{s}$-bounded.

In what follows we show that some positive results can also be found on the relation among extreme points and right boundedness.

Other results on the behavior of asymmetric norms in finite dimensional spaces can be proven using the tools developed above.

Lemma 5.2. For any finite dimensional asymmetric normed space $(X, q)$, we always have $B_{1}^{q}[0]=S\left(B_{1}^{q}[0]\right)+\theta_{q}$. 
Proof. Using a well-known result of Klee [17], we know that $B_{1}^{q}[0]$ is the convex hull of its extreme points and its extreme rays? This implies that $S\left(B_{1}^{q}[0]\right) \subset B_{1}^{q}[0]$ and therefore

$$
S\left(B_{1}^{q}[0]\right)+\theta_{q} \subset B_{1}^{q}[0]+\theta_{q}=B_{1}^{q}[0] .
$$

In order to prove the other contention, let $x \in B_{1}^{q}[0]$. By [16, Lemma 3.6] every extreme ray $B_{1}^{q}[0]$ is parallel to some ray contained in $\theta_{q}$. Then we can find $a_{1}, \ldots, a_{n}, a_{n+1}, \ldots, a_{p} \in E\left(B_{1}^{q}[0]\right), b_{1}, \ldots, b_{n} \in \theta_{q}$, and scalars $\lambda_{1}, \ldots, \lambda_{p}, t_{1}, \ldots, t_{n} \geq 0$ such that

$$
x=\sum_{i=1}^{n} \lambda_{i}\left(a_{i}+t_{i} b_{i}\right)+\sum_{i=n+1}^{p} \lambda_{i} a_{i} \quad \text { and } \quad \sum_{i=1}^{p} \lambda_{i}=1 .
$$

Since $\theta_{q}$ is a convex cone, the point $\sum_{i=1}^{n} \lambda_{i} t_{i} b_{i}$ lies in $\theta_{q}$. On the other hand, we know that $\sum_{i=1}^{p} \lambda_{i} a_{i} \in S\left(B_{1}^{q}[0]\right)$. Thus

$$
\begin{aligned}
x & =\sum_{i=1}^{n} \lambda_{i}\left(a_{i}+t_{i} b_{i}\right)+\sum_{i=n+1}^{p} \lambda_{i} a_{i} \\
& =\sum_{i=1}^{p} \lambda_{i} a_{i}+\sum_{i=1}^{n} \lambda_{i} t_{i} b_{i} \\
& \in S\left(B_{1}^{q}[0]\right)+\theta_{q} .
\end{aligned}
$$

Proposition 5.3. Let $(X, q)$ be an asymmetric normed space such that $E\left(B_{1}^{q}[0]\right)$ is $q^{s}$-bounded. Then $q$ is right bounded.

Proof. If $E\left(B_{1}^{q}[0]\right)$ is $q^{s}$-bounded, so is $S\left(B_{1}^{q}[0]\right)$. Thus, by Lemmas 2.4 and 5.2 we conclude that $q$ is right bounded.

The converse of Proposition 5.3 is false. A counterexample of this situation was already shown in Example 4.1, where the norm is right bounded, but the set of extreme points of $B_{1}^{q}[0]$ is not $q^{s}$-bounded.

However, if the norm is 1-bounded, the situation is different as we can see in the following

Proposition 5.4. Let $(X, q)$ be a finite dimensional 1-bounded asymmetric normed space. Then $E\left(B_{1}^{q}[0]\right) \subset B_{1}^{q^{s}}[0]$ and therefore $E\left(B_{1}^{q}[0]\right)$ is $q^{s}$ bounded.

Proof. Since $q$ is 1-bounded, the set $B_{1}^{q}[0]=B_{1}^{q^{s}}[0]+\theta_{0}$. Since $B_{1}^{q^{s}}[0]$ is $q^{s}$-compact (and $q$-compact), we can use [16, Theorem 4.1] to infer that $E\left(B_{1}^{q}[0]\right) \subset B_{1}^{q^{s}}[0]$ as desired.

\footnotetext{
${ }^{2}$ An open half line $R=\{a+t b \mid a, b \in X, t>0\}$ is called an extreme ray of $A$ if $y, z \in R$ whenever $\lambda y+(1-\lambda) z \in R$, where $y, z \in A$ and $\lambda \in(0,1)$. In this case, if $R$ is an extreme ray and the extreme $a$ lies in $A$, then $a$ is an extreme point of $A$.
} 
Let us finish the paper with the following open question, that is suggested by the strong relation between right boundedness and local compactness.

Question 5.5. Let $(X, q)$ be a finite dimensional asymmetric normed space. If $X$ is locally compact, is $q$ right bounded? In particular, if $B_{1}^{q}[0]$ is compact, is $q$ right bounded?

\section{REFERENCES}

[1] C. Alegre, J. Ferrer, and V. Gregori, On the Hahn-Banach theorem in certain linear quasi-uniform structures, Acta Math. Hungar. 82 (1999), 315-320.

[2] C. Alegre, I. Ferrando, L. M. García Raffi, and E. A. Sánchez Pérez, Compactness in asymmetric normed spaces, Topology Appl. 155 (2008), 527-539.

[3] C. D. Aliprantis and K. Border, Infinite Dimensional Analysis. A Hitchiker's Guide, 3th, edition, Springer, Berlin Heidelberg, 2006.

[4] C. Bessaga, and A. Pełczyński, Selected topics in infinite-dimensional topology. PWN - Polish Scientific Publishers, Warszawa, 1975.

[5] P. A. Borodin, The Banach-Mazur theorem for spaces with asymmetric norm and its applications in convex analysis, Mathematical Notes, 69 (3) (2001), 298-305.

[6] S. Cobzaş, Separation of convex sets and best approximation in spaces with asymmetric norm, Quaest. Math. 27(3) (2004), 275-296.

[7] S. Cobzaş, Functional analysis in asymmetric normed spaces, Birkhäuser. Basel. 2013.

[8] J. Conradie and M. D. Mabula, Completeness, precompactness and compactness in finite-dimensional asymmetrically normed lattices, Topology Appl. 160 (2013), 20122024.

[9] J. Conradie, Asymmetric norms, cones and partial orders. Topology Appl. 193 (2015), 100-115.

[10] L. M. García-Raffi, S. Romaguera, and E. A. Sánchez-Pérez, Sequence spaces and asymmetric norms in the theory of computational complexity. Math. Comp. Model. 36 (2002), 1-11.

[11] L. M. García Raffi, S. Romaguera, and E. A. Sánchez Pérez, The bicompletion of an asymmetric normed linear space, Acta Math. Hungar. 97 (3) (2002), 183-191.

[12] L. M. García Raffi, S. Romaguera, and E. A. Sánchez Pérez, On Hausdorff asymmetric normed linear spaces, Houston J. Math. 29 (2003), 717-728.

[13] L. M. García Raffi, S. Romaguera, and E. A. Sánchez-Pérez, The dual space of an asymmetric normed linear space, Quaest. Math. 26 (2003), 83-96.

[14] L. M. García Raffi, Compactness and finite dimension in asymmetric normed linear spaces, Topology Appl. 153 (2005), 844-853.

[15] N. Jonard-Pérez, E. A. Sánchez-Pérez, Compact convex sets in 2-dimensional asymmetric normed spaces, Quaest. Math. 39(1) (2016), 73-82.

[16] N. Jonard-Pérez and E. A. Sánchez-Pérez, Extreme points and geometric aspects of compact convex sets in asymmetric normed spaces, Topology Appl. 203 (2016), 12-21.

[17] V. L. Klee, Extremal structure of convex sets, Arch. Math. 8 (3) (1957), 234-240.

[18] R. Webster, Convexity, Oxford University Press, New york, 1994.

[Natalia Jonard-Pérez] Departamento de Matemáticas, Facultad de Ciencias, Universidad Nacional Autónoma de México. Circuito Exterior S/N, Cd. 
Universitaria, Colonia Copilco el Bajo, Delegación Coyoacán, 04510, México D.F., México, e-mail: nat@ciencias.unam.mx

[Enrique A. Sánchez Pérez] Instituto Universitario de Matemática Pura y Aplicada, Universitat Politècnica de València, Camino de Vera s/n, 46022 Valencia, Spain, e-mail: easancpe@mat.upv.es 